\title{
Corrigendum
}

\section{Geographical inequalities in lung cancer management and survival in South East England: evidence of variation in access to oncology services?}

\author{
RH Jack, MC Gulliford, J Ferguson and H Møller
}

British Journal of Cancer (2004) 91, I852. doi: I0. I038/sj.bjc.6602247 www.bjcancer.com

(c) 2004 Cancer Research UK

Correction to: British Journal of Cancer (2003) 88, 1025-1031. doi: 10.1038/sj.bjc.6600831

The authors have recently notified us of an error in the above paper. It had come to their attention that, due to a coding error, a number of patients who received chemotherapy or radiotherapy were misclassified as having no treatment. The proportions of patients receiving any chemotherapy, radiotherapy and any active treatment were too low as reported. The median percentage and range of patients receiving different treatments within the health authorities are shown below in the revised Table 3.

In the revised analyses, there was still evidence of health authority level variation in treatment and survival similar to that shown in Figure 1. The associations of area and health service characteristics are shown in the revised Table 5. There were no material changes in the associations of survival with all area and health service characteristics. In the analysis of determinants of chemotherapy, radiotherapy and any treatment, the associations of

Table 3 (revised) Median percentage and range of lung cancer patients with each treatment by health authority, 1995-1999

\begin{tabular}{|c|c|c|}
\hline & Med \% & Range \\
\hline None registered ${ }^{a}$ & 38 & $32-55$ \\
\hline Chemotherapy only (Chem) & 10 & $6-17$ \\
\hline Radiotherapy only (RT) & 32 & $25-44$ \\
\hline Non-investigative surgery only (S) & 6 & $4-10$ \\
\hline Chem+RT & 9 & $5-14$ \\
\hline S+Chem & 0 & $0-1$ \\
\hline$S+R T$ & 1 & $0-3$ \\
\hline $\mathrm{S}+\mathrm{Chem}+\mathrm{RT}$ & 0 & $0-1$ \\
\hline Investigative surgery only & 20 & $15-40$ \\
\hline Any Chem & 20 & $14-29$ \\
\hline Any RT & 42 & $31-54$ \\
\hline Any $\mathrm{S}$ & 7 & $5-12$ \\
\hline Any treatment ${ }^{b}$ & 62 & $45-68$ \\
\hline First hospital visited a radiotherapy centre & 27 & $8-79$ \\
\hline
\end{tabular}

ancludes patients who had investigative surgery only. ${ }^{\mathrm{b}}$ Any treatment $=$ any chemomerapy, radiotherapy or non-investigative surgery. treatment with the lung cancer incidence rates were sensitive to correction of the error.

The main conclusion of the paper that there is significant geographical variation in the treatment of lung cancer, which may be associated with differential access to oncology services, is still supported.

Table 5 (revised) Area and organisational characteristics associated with treatment and survival

\begin{tabular}{|c|c|c|c|}
\hline & OR & $95 \% \mathrm{Cl}$ & $P$-value \\
\hline \multicolumn{4}{|l|}{ Any treatment } \\
\hline First hospital visited a radiotherapy centre & 1.84 & $1.46-2.32$ & $<0.000$ \\
\hline Deprivation (Townsend score) & 0.98 & $0.96-0.99$ & 0.0091 \\
\hline Age standardised incidence (per 100 000) & 1.00 & $0.99-1.01$ & 0.9128 \\
\hline \multicolumn{4}{|l|}{ Any chemotherapy } \\
\hline First hospital visited a radiotherapy centre & 1.24 & $0.98-1.55$ & 0.0699 \\
\hline Deprivation (Townsend score) & 0.96 & $0.94-0.98$ & 0.0001 \\
\hline Age standardised incidence (per 100000 ) & 1.02 & $1.01-103$ & 0.0018 \\
\hline \multicolumn{4}{|l|}{ Any radiotherapy } \\
\hline First hospital visited a radiotherapy centre & 1.67 & $1.32-2.10$ & $<0.0001$ \\
\hline Depris & 1.00 & $0.99-1.02$ & 0.7759 \\
\hline Age standardised incidence (per 100000 ) & 1.00 & $0.99-1.01$ & 0.9748 \\
\hline \multicolumn{4}{|l|}{ Any non-investigate surgery } \\
\hline First hospital visited a radiotherapy centre & 1.02 & $0.63-1.64$ & 0.9383 \\
\hline Deprivation (Townsend score) & 0.98 & $0.95-1.01$ & 0.1206 \\
\hline Age standardised incidence (per 100000 ) & 0.97 & $0.96-0.99$ & 0.0005 \\
\hline \multicolumn{4}{|l|}{ I-year sunvival ${ }^{\mathrm{a}}$} \\
\hline First hospital visited a radiotherapy centre & 1.18 & $0.96-1.45$ & 0.1146 \\
\hline Deprivation (Townsend score) & 1.01 & $1.00-1.03$ & 0.0827 \\
\hline Age standardised incidence (per 100000 ) & 0.99 & $0.98-1.00$ & 0.0208 \\
\hline \multicolumn{4}{|l|}{ 3-year sunvival ${ }^{\mathrm{a}}$} \\
\hline First hospital visited a radiotherapy centre & 1.19 & $0.99-1.43$ & 0.0674 \\
\hline Deprivation (Townsend score) & 1.01 & $1.00-1.03$ & 0.1320 \\
\hline Age standardised incidence (per 100000 ) & 0.99 & $0.98-1.00$ & 0.0276 \\
\hline
\end{tabular}

Odds ratios adjusted for sex, age group, histology and stage of tumour, deprivation, lung cancer incidence, whether first hospital attended was a radiotherapy centre and

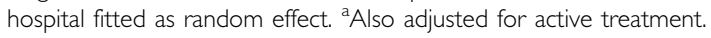

